\title{
Integrated metabolome and transcriptome revealed the flavonoid biosynthetic pathway in developing Vernonia amygdalina leaves
}

\author{
Lanya Shui ${ }^{\text {Equal first author, } 1}$, Kaisen Huo ${ }^{\text {Equal first author, } 1}$, Yan Chen ${ }^{1}$, Zilin Zhang ${ }^{1}$, Yanfang Li ${ }^{1}$, Jun Niu ${ }^{\text {Corresp. } 1}$ \\ ${ }^{1}$ College of Forestry, Hainan University, Haikou, HN, China \\ Corresponding Author: Jun Niu \\ Email address: niujun@hainanu.edu.cn
}

Background : Vernonia amygdalina as a tropical horticultural crop has been widely used for medicinal herb, feed, and vegetable. Recently, increasing studies revealed that this species possesses multiple pharmacological properties. Notably, $V$. amygdalina leaves possess an abundance of flavonoids, but the specific profiles of flavonoids and the mechanisms of $f$ avonoid bi osynthesis in developing leaves are largely unknown.

Methods: The total flavonoids of $V$. amygdalina leaves were detected using ultraviolet spectrophotometer. The temporal flavonoid profiles of $V$. amygdalina leaves were analyzed by LC-MS. The transcriptome analysis of $V$. amygdalina leaves was performed by Illumina sequencing. Functional annotation and differential expression analysis of $V$. amygdalina genes were performed by Blast2GO V2.3.5 and RSEM V1.2.31, respectively. qRT-PCR analysis was used to verify the gene expressions in developing $V$. amygdalina leaves.

Results: By LC-MS analysis, three substrates ( $p$-coumaric acid, trans-cinnamic acid, and phenylalanine) for flavonoid biosynthesis were identified in V. amygdalina leaves. Additionally, 42 flavonoids were identified from $V$. amygdalina leaves, including 6 dihydroflavones, 14 flavones, 8 isoflavones, 9 flavonols, 2 xanthones, 1 chalcone, 1 cyanidin, and 1 dihydroflavonol. Glycosylation and methylation were common at the hydroxy group of C3, C7, and C4' positions. Moreover, dynamic patterns of different flavonoids showed diversity. By Illumina sequencing, the obtained over 200 million valid reads were assembled into 60,422 genes. Blast analysis indicated that 31,872 genes were annotated at least in one of public databases. Greatly increasing molecular resources make up the lack of gene information in $V$. amygdalina. By digital expression profiling and qRT-PCR, we specifically characterized some key enzymes, such as Va-PAL1, Va-PAL4, Va-C4H1, Va-4CL3, Va-ACC1, Va-CHS1, Va-CHI, Va-FNSII, and VaIFS3, involved in flavonoid biosynthesis. Importantly, integrated metabolome and transcriptome data of $V$. amygdalina leaves, we systematically constructed a flavonoid biosynthetic pathway with regards to material supplying, flavonoid scaffold biosynthesis, and flavonoid modifications. Our findings contribute significantly to understand the underlying mechanisms of flavonoid biosynthesis in $V$. amygdalina leaves, and also provide valuable information for potential metabolic engineering. 


\title{
1 Integrated metabolome and transcriptome revealed
}

2 the flavonoid biosynthetic pathway in developing

3 Vernonia amygdalina leaves

\author{
4 Lanya Shui ${ }^{1 \dagger}$, Kaisen Huo ${ }^{1 \dagger}$, Yan Chen ${ }^{1}$, Zilin Zhang ${ }^{2}$, Yanfang Li², Jun Niu ${ }^{*}$,
}

5

6

7

8

9

10

11

12

1 Key Laboratory of Genetics and Germplasm Innovation of Tropical Special Forest Trees and Ornamental Plants, College of Forestry, Hainan University, Haikou, Hainan 570228, China.

†Equal contributors

*Corresponding author: +86-898-66268107, niujun@hainanu.edu.cn

Corresponding Author:

Jun Niu

58 Renmin Dadao, Hainan University, Haikou, Hainan 570228, China.

Email address: niujun@hainanu.edu.cn

\section{Abstract}

Background: Vernonia amygdalina as a tropical horticultural crop has been widely used for medicinal herb, feed, and vegetable. Recently, increasing studies revealed that this species possesses multiple pharmacological properties. Notably, V. amygdalina leaves possess an abundance of flavonoids, but the specific profiles of flavonoids and the mechanisms of flavonoid biosynthesis in developing leaves are largely unknown.

Methods: The total flavonoids of $V$. amygdalina leaves were detected using ultraviolet spectrophotometer. The temporal flavonoid profiles of $V$. amygdalina leaves were analyzed by LC-MS. The transcriptome analysis of $V$. amygdalina leaves was performed by Illumina sequencing. Functional annotation and differential expression analysis of $V$. amygdalina genes were performed by Blast $2 \mathrm{GO}$ v2.3.5 and RSEM v1.2.31, respectively. qRT-PCR analysis was used to verify the gene expressions in developing $V$. amygdalina leaves.

Results: By LC-MS analysis, three substrates ( $p$-coumaric acid, trans-cinnamic acid, and phenylalanine) for flavonoid biosynthesis were identified in V. amygdalina leaves. Additionally, 42 flavonoids were identified from $V$. amygdalina leaves, including 6 dihydroflavones, 14 flavones, 8 isoflavones, 9 flavonols, 2 xanthones, 1 chalcone, 1 cyanidin, and 1 dihydroflavonol. Glycosylation and methylation were common at the hydroxy group of C3, C7, and C4' positions. Moreover, dynamic patterns of different flavonoids showed diversity. By Illumina sequencing, the obtained over 200 million valid reads were assembled into 60,422 genes. Blast analysis indicated that 31,872 genes were annotated at least in one of public databases. Greatly increasing molecular resources make up the lack of gene information in $V$. amygdalina. By digital expression profiling and qRT-PCR, we specifically characterized some key enzymes, such as Va-PAL1, Va-PAL4, Va-C4H1, Va-4CL3, Va-ACC1, VaCHS1, Va-CHI, Va-FNSII, and Va-IFS3, involved in flavonoid biosynthesis. Importantly, integrated metabolome and transcriptome data of $V$. amygdalina leaves, we systematically constructed a flavonoid biosynthetic pathway with regards to material supplying, flavonoid scaffold biosynthesis, and flavonoid modifications. Our findings contribute significantly to understand the underlying mechanisms of 
42 flavonoid biosynthesis in $V$. amygdalina leaves, and also provide valuable information for potential

43 metabolic engineering.

44

45

46

47

48

49

50

51

52

53

54

55

56

57

58

59

60

61

62

63

64

65

66

67

68

69

70

71

72

73

74

75

76

77

78

79

80

81

82

83

84

85

\section{Introduction}

Vernonia amygdalina, belonging to family Asteraceae, is a rapidly regenerating shrub (Yeap et al. 2010). This plant is widely distributed in the humid tropical forest of Sub-saharan Africa, Southeast Asia, and southern coastal regions of China (Igile et al. 1994). Historically, V. amygdalina has been planted as a tropical horticultural crop widely used for medicinal herb, feed, and vegetable in Asia and Africa.

Recently, increasing studies revealed that this species possesses multiple pharmacological properties, including antibacterial, antifungal, antiparasite, antimalaria, antihelmintic, antiviral, anticancer, antimutagentic, and antidiabetic activities (Alara et al. 2020; Asante et al. 2016; Atangwho et al. 2013; Erasto et al. 2007; Yeap et al. 2010). Thus, phytochemical analysis of $V$. amygdalina has been extensively concerned in the academic field. Notably, sesquiterpenoid lactones, terpenoids, flavonoids, saponins, steroids glycosides, anthraquinone, and coumarins have been characterized in this species (Ayoola et al. 2008; Igile et al. 1994; Nwanjo 2005; Tona et al. 2004).

Flavonoids are classified as plant-specific secondary metabolite, consisting of two phenolic rings linked with one oxane ring (C6-C3-C6). Numerous studies have shown that flavonoids have various biofunctions in plants, such as phototropic response (Silva-Navas et al. 2016), protection against ultraviolet-B, cold stress, herbivores, and pathogens (Dixon \& Pasinetti 2010). Since the objectively pharmacological activities of flavonoids against many chronic diseases, including neurodegenerative and cancer diseases (Tohge \& Fernie 2017), studies of flavonoids have gained popular attention. Interestingly, phytochemical screening of $V$. amygdalina showed abundant flavonoids in leaves, and thus this species is considered as one of natural source of flavonoids (Alara et al. 2018; Asante et al. 2016).

It is well known that $p$-coumaroyl-CoA and malonyl-CoA are initial substrates for flavonoid biosynthesis in the cytoplasm. First, as the first rate-limiting enzyme in phenylalanine pathways, phenylalanine ammonia-lyase (PAL) converts phenylalanine into trans-cinnamic acid, which is involved in the lignin, lignan, and flavonoid synthesis (Maeda \& Dudareva 2012). Then, cinnamic acid 4hydroxylase $(\mathrm{C} 4 \mathrm{H})$ hydroxylates trans-cinnamic acid at the $\mathrm{C} 4$ position to produce $p$-coumaric acid (4coumaric acid) (Schilmiller et al. 2009). Last, 4-coumaric acid: CoA ligase (4CL) that is an ATPdependent enzyme irreversibly catalyzes the $p$-coumaroyl-CoA production (Hamberger \& Hahlbrock 2004). In plant, acetyl-CoA is critical material for the biosynthesis of many metabolites. ATP-citrate lyase (ACL) produces the cytoplasmic pool of acetyl-CoA, which can be carbonylated into malonyl-CoA by acetyl-CoA carboxylase (ACC). Compared with plastidial ACC comprising four distinct subunits, cytoplasmic ACC is composed of a large polypeptide with four functional domains, participating in malonyl-CoA formation for flavonoid biosynthesis in cytoplasm (Yanai et al. 1995).

When malonyl-CoA and $p$-coumaroyl-CoA have been formed, flavonoid scaffolds can be synthesized by a series of regulatory enzymes (Saito et al. 2013). As the first rate-limiting enzyme in the biosynthesis pathway of flavonoids, chalcone synthase (CHS) condenses one molecule of $p$-coumaroyl-CoA and three molecules of malonyl-CoA into naringenin chalcone (Austin \& Noel 2003). Subsequently, naringenin chalcone can be stereospecifically cyclized to (2S)-naringenin by chalcone isomerase (CHI) (Lepiniec et al. 2006). It is worth noting that the generated (2S)-naringenin can be converted into different flavonoid kinds by different regulatory enzymes. For example, flavone synthase II (FNSII) functions in the first step of the trunk pathway towards flavones (Ferreyra et al. 2015). Isoflavone synthase (IFS) catalyzes the isomerization of (2S)-naringenin into the isoflavone biosynthesis (Jung et al. 2000). Additionally, 
86

87

88

89

90

91

92

93

94

95

96

97

98

99

100

101

102

103

104

105

106

107

108

109

110

111

112

113

114

115

116

117

118

119

120

121

122

123

124

125

126

127

128

flavanone 3-hydroxylase ( $\mathrm{F} 3 \mathrm{H})$ catalyzes the oxygenation at C3-postion of (2S)-naringenin to form dihydroflavonols (Pelletier \& Shirley 1996b). Together, the biosynthesis pathway of flavonoid scaffolds is generally conserved across plant species (Tohge et al. 2017).

Despite molecular structures of flavonoid scaffolds in different plants are restricted (Li et al. 2020; Liu et al. 2020), multi-terminal modifications of flavonoids, catalyzed by glycosyltransferase, methyltransferase, phenylacyltransferase, and acyltransferase, result in the chemical diversity of flavonoids (Saito et al. 2013). These tailoring modifications affect the biological roles of flavonoids in plants. For example, glycosylation is responsible for the steady accumulation of flavonoids ( $\mathrm{Li}$ et al. 2001) and phenylacetylation enhances UV-B absorbent properties (Tohge et al. 2016).

In the current study, to confirm the qualitative and quantitative flavonoid profiles in developing $V$. amygdalina leaves, the leaf samples collected from 10,20 and 30 days after germination (DAG) were analyzed by LC-MS. Subsequently, the transcriptomic sequencing was perform using Illumina technology. After functional annotation, the genes involved in malonyl-CoA formation, phenylpropanoid pathway, flavonoid biosynthesis, and tailoring reactions of flavonoids were characterized in $V$. amygdalina leaves. Combined with digital expression analysis and qRT-PCR results, the expressed patterns of some key enzymes associated with flavonoid biosynthesis were constructed. Overall, the regulatory enzymes involved in flavonoid biosynthesis were systematically analyzed in developing $V$. amygdalina leaves, which will contribute to the targeted and artificial regulation of specific flavonoid biosynthesis.

\section{Materials \& Methods}

\section{Plant material}

Five plants located in the plantation base of Hainan University (geographical coordinates 110.329463 E; $20.062906 \mathrm{~N}$ ) were selected. Based on our previous investigation on the developmental process of $V$. amygdalina leaves, the leaves grow rapidly in size before $30 \mathrm{DAG}$, and then enter the mature stage with slow growth or on change before senescence. Therefore, the leafbuds were marked and the leaves were collected from $10 \mathrm{DAG}, 20 \mathrm{DAG}$ and $30 \mathrm{DAG}$, representing the different developmental phares. Five leaves from each plant were selected each time. The fresh leaves were washed with distilled water and dried, and then were rapidly frozen in liquid nitrogen and stored at $-80^{\circ} \mathrm{C}$ until use.

\section{Extraction and determination of total flavonoids}

$5 \mathrm{~g}$ of fresh $V$. amygdalina leaves were ground into powder and extracted by $70 \mathrm{~mL} 95 \%$ ethanol for 2 h. After filtration, liposoluble substances were removed by solvent extraction with $35 \mathrm{~mL}$ petroleum ether. The above $10 \mathrm{~mL}$ extracted lipid was added into a $25 \mathrm{~mL}$ volumetric flask. The $2.5 \mathrm{~mL} 30 \%$ ethanol and $0.75 \mathrm{~mL} 5 \%$ sodium nitrite solution were added into volumetric flask, mixing and standing for $5 \mathrm{~min}$. Then, $0.75 \mathrm{~mL} 10 \%$ aluminum nitrate solution was added, mixing and standing for $5 \mathrm{~min}$. Subsequently, $10 \mathrm{~mL} 1 \mathrm{~mol} / \mathrm{L} \mathrm{NaOH}$ solution was added and agitated. Last, dilute with $30 \%$ ethanol to volume, incubating for $10 \mathrm{~min}$. Three biological repetitions were performed. The $10 \mathrm{~mL}$ extracted lipid was added into a $25 \mathrm{~mL}$ volumetric flask, and rutin (Solarbio HPLC $>98 \%$ ) was used as standard for manufacturing a standard curve. Ultraviolet spectrophotometer (Persee: TU-1810) was used to set the wavelength at $510 \mathrm{~nm}$ to measure the absorbance.

\section{Metabolite extraction}

Leaf samples were ground in liquid nitrogen. The $50 \mathrm{mg}$ sample was immediately transferred in an EP tube with $1 \mathrm{~mL} \mathrm{MeOH}: \mathrm{CAN}: \mathrm{H}_{2} \mathrm{O}(4: 4: 2, \mathrm{~V} / \mathrm{V})$ at $-20^{\circ} \mathrm{C}$ precooling. Then, eddy oscillated for $1 \mathrm{~min}$ and ultrasonic treated for $40 \mathrm{~min}$ at $4^{\circ} \mathrm{C}$. The mixture was incubated at $20^{\circ} \mathrm{C} 120 \mathrm{~min}$ and centrifuged at $4^{\circ} \mathrm{C}$

Peer) reviewing PDF | (2020:11:55707:2:0:NEW 8 Mar 2021) 
129

130

131

132

133

134

135

136

137

138

139

140

141

142

143

144

145

146

147

148

149

150

151

152

153

154

155

156

157

158

159

160

161

162

163

164

165

166

167

168

169

170

171

172

$17000 \mathrm{~g}$ for $15 \mathrm{~min}$. The $200 \mu \mathrm{L}$ supernatant was transferred into a new EP tube, and dried in a vacuum at $35^{\circ} \mathrm{C}$. The sample was dissolved in $400 \mu \mathrm{L}$ acetonitrile: $\mathrm{ddH} 2 \mathrm{O}(1: 1, \mathrm{v} / \mathrm{v})$, vortex for $60 \mathrm{sec}$ and filtrate with $0.22 \mu \mathrm{m}$ organic membrane. $120 \mu \mathrm{L}$ supernatant was transferred to a sample bottle for LC-MS analysis. The extraction was replicated three times.

\section{LC-MS analysis}

For metabonomic analysis, the extracted samples were analyzed using Agilent 1290 equipped with ACQUITY UPLC HSS T3 $1.8 \mu \mathrm{m} 2.1 \times 100 \mathrm{~mm}$ (Waters). The parameters are described as follows: column temperature, $35^{\circ} \mathrm{C}$; injection volume, $1 \mu \mathrm{L}$; solvent $\mathrm{A}$ (ddH2O: formic acid, 1000:1, v/v) and solvent B (CH3CN: formic acid, 1000:1, v/v) for positive ion mode; solvent A (2mM ammonium formate) and solvent B (100\% CH3CN) for negative ion mode; flow rate $400 \mu \mathrm{L} / \mathrm{min}$, gradient: 0.0 - $0.5 \mathrm{~min}, 95 \%$ solvent A; 0.5 - $2.0 \mathrm{~min}, 95-90 \%$ solvent A; 2.0 - $10.0 \mathrm{~min}, 90-40 \%$ solvent A; 10.0 - $14.0 \mathrm{~min}, 40-5 \%$ solvent A, 14.0 - $16.0 \mathrm{~min}, 5 \%$ solvent A, 16.0 - $18.0 \mathrm{~min}, 5-95 \%$ solvent A, 18.0 - $20.0 \mathrm{~min}, 95 \%$ solvent A. MS data were obtained by Agilent 6545QTOF (California, USA). The parameters were as follows: mass scan range $50-1100 \mathrm{~m} / \mathrm{z}$; gas temperature $320^{\circ} \mathrm{C}$; gas flow $8 \mathrm{~L} / \mathrm{min}$; sheath gas flow $12 \mathrm{~L} / \mathrm{min}$; sheath gas temp $320^{\circ} \mathrm{C}$; VCap $3500 \mathrm{~V}$ (negative) and $4000 \mathrm{~V}$ (positive). MSDIAL3.08 software was used to analyze our data.

\section{cDNA library sequencing method and process}

Total RNA was extracted from samples using RNeasy Plant Mini Kits (Qiagen). mRNA was enriched with Oligo (dT) magnetic beads, and fragmented by adding fragmentation buffer. The fragmented mRNA was used as template to synthesize the first and second cDNA strand with random hexamers. After purification by QiaQuick PCR kit (Qiagen), the poly (A) and sequencing sequence were added. After selection by agarose gel electrophoresis and amplification by PCR, the established cDNA library was sequenced by Illumina HiSeqTM. To ensure data quality, clean reads were obtained by filtering the original data. All transcriptomic data can be accessed in BioProject PRJNA554198. Trinity v2.2.0 software was used for de novo transcriptome assembly. The sequencing was replicated three times for each sample.

\section{Functional annotation and differential expression analysis}

The assembled genes were annotated against the following public databases, TAIR10, Nr (NCBI nonredundant protein sequences), Swiss-Prot and COG (Clusters of Orthologous Groups of proteins) by Blast+ v2.4.0 (E-value $\left.<1 \mathrm{e}^{-5}\right)$. Gene Ontology (GO) functional classifications and Kyoto Encyclopedia of Genes and Genomes (KEGG) assignments were performed by Blast2GO v2.3.5 (Götz et al. 2008) and KOBAS software (Kanehisa et al. 2008), respectively. Transcriptome assembly completeness were assessed using the 2,326 conserved genes of eudicots in BUSCO v4.1.2 (Simao,2015) with the BLAST Evalue cutoff set to 0.001 (Simão et al. 2015).

The expressed levels of genes were estimated using Fragment Per Kilobase of exon model per Million mapped reads (FPKM) by RSEM v1.2.31. Differential expression genes (DEGs) between two groups were performed using edgeR v3.14.0. Genes with false discovery rate (FDR) $<0.01$ and $\left|\log _{2} \mathrm{FC}\right|>1$ were assigned as DEGs.

\section{qRT-PCR analysis}

The above total RNA was used and reversely transcribed using the Reverse Transcription System (Promega). Using PrimerQuest (www.idtdna.com/PrimerQuest/Home/Index), the qRT-PCR were designed (Supplementary Table S1). According to our transcriptome results, 10 housekeeping genes with stable expression levels were selected for stability analysis. Using NormFinder approach, actin-related protein subunit 3 was regarded as the best stable reference in developing $V$. amygdalina leaves 
173 (Supplementary Figure S1). The relative expression levels were calculated as $\log _{2}\left((1+\mathrm{E} 1)^{\Delta \mathrm{Ct} 1(\mathrm{Control}-}\right.$

174 Sample) $\left./(1+\mathrm{E} 2)^{\Delta \mathrm{Ct} 2(\mathrm{Control}-S a m p l e)}\right), \mathrm{E} 1$ : PCR efficiency of target-gene primer; E2: PCR efficiency of

175 reference-gene primer; $\Delta \mathrm{Ct} 1$ : the difference of $\mathrm{Ct}$ value between control and sample in experimental

176 group; the difference of $\mathrm{Ct}$ value between control and sample in reference group. The PCR efficiency (E)

177 was estimated from the data obtained from the exponential phase of each individual amplification plot

178 and the equation $\left(\mathrm{E}=10^{\text {slope }}\right)$ (Ramakers et al. 2003). The expression levels were analyzed from four

179 biological replicates.

180 Statistical analysis

181

182

The correlation analysis between the relative contents of flavonoids and gene expressions was

183

preformed using Pearson method. Differences between samples were tested for statistical significance using the Duncan MRT method. Statistical analysis was implemented by SPSS software (version 19.0).

184

185

186

187

188

189

190

191

192

193

194

195

196

197

198

199

200

201

202

203

204

205

206

207

208

209

210

211

212

213

214

215

\section{Results}

\section{The total content of flavonoids in developing $V$. amygdalina leaves}

To evaluate flavonoid profiles in developing $V$. amygdalina leaves, the samples were collected at early (10 DAG), middle (20 DAG), and late (30 DAG) development. It was obvious that the leaf size was increased in proportion to the degree of development, for example width ranged from $2.84 \pm 0.29 \mathrm{~cm}$ to $5.65 \pm 0.33 \mathrm{~cm}$, and length ranged from $7.28 \pm 0.12 \mathrm{~cm}$ to $12.48 \pm 0.11 \mathrm{~cm}$ (Figure 1a). Preliminary extraction revealed the presence of flavonoids in developing $V$. amygdalina leaves. The resulting $2.20 \%$, $2.52 \%$, and $1.81 \%$ of flavonoid content were identified at 10, 20, and $30 \mathrm{DAG}$, respectively (Figure 1b).

Compared with mature leaves at $30 \mathrm{DAG}$, these data indicated a significant accumulation of flavonoids at early-middle (10-20 DAG) development of $V$. amygdalina leaves.

\section{Flavonoid profiles for $\boldsymbol{V}$. amygdalina leaves}

By LC-MS analysis, $p$-coumaric acid, trans-cinnamic acid, and phenylalanine involved in transformation pathway of substrate for flavonoid biosynthesis were identified in $V$. amygdalina leaves. The quantitative analysis indicated that phenylalanine and $p$-coumaric acid displayed a similar dynamic pattern, a gradual decline during developing $V$. amygdalina leaves (Supplementary Table S2 and Figure 2). trans-cinnamic showed a significantly highest relative content at $10 \mathrm{DAG}$ (Supplementary Table S2 and Figure 2).

A total of 42 flavonoids were obtained in $V$. amygdalina leaves by LC-MS analysis, including 6 dihydroflavones, 14 flavones, 8 isoflavones, 9 flavonols, 2 xanthones, 1 chalcone, 1 cyanidin, and 1 dihydroflavonol (Supplementary Table S2). It was observed that diosmetin, nepetin, and luteolin accounted for a relatively large percentage (Supplementary Table S2), implying that the major flavonoid component in $V$. amygdalina leaves is flavones.

Moreover, the dynamic patterns of different flavonoids showed diversity during leaf development (Figure 2). For example, nepetin, isorhamnetin, and eriodictyol were preferentially accumulated at 10 DAG, diosmetin, genistein, and prunetin at $20 \mathrm{DAG}$, and baicalin, tectorigenin, and 7-hydroxyflavonoids at $30 \mathrm{DAG}$ (Supplementary Table S2). Intriguingly, some flavonoids were specially accumulated at specific period, such as naringenin, apigenin, apigetrin, orientin, ononin, and kaempferol-3-glucuronide at $10 \mathrm{DAG}, 6,8$-diprenylnaringenin and biochanin A- $\beta$-D-glucoside at $20 \mathrm{DAG}$, and isoflavonoid Oglycosides and taxifolin at $30 \mathrm{DAG}$ (Figure 2). These variant favonoid profiles suggest that they may have different functions in $V$. amygdalina leaves.

Importantly, most of flavonoids involved in formation of flavonoid scaffolds were identified in this work, including naringenin chalcone, naringenin, eriodictyol, apigetrin, luteolin, genistein, taxifolin, and 
216 kaempferol (Supplementary Table S2). In addition, it was observed that flavonoid glycosylation and

217 methylation were common in $V$. amygdalina leaves, and generally occurred at the hydroxy group of $\mathrm{C} 3$,

218 C7, and C4' positions of flavonoid aglycones, such as Pratol, Diosmetin, Baicalin, Prunetin, kaempferol

219 7-O- $\beta$-D-glucopyranoside, Biochanin A- $\beta$-D-glucoside, Tiliroside, and Kaempferol-3-Glucuronide

220 (Supplementary Table S2).

221

222

223

224

225

226

227

228

229

230

231

232

233

234

235

236

237

238

239

240

241

242

243

244

245

246

247

248

249

250

251

252

253

254

255

256

257

258

\section{Transcriptome sequencing and gene assembly}

To obtain accurate gene data in developing $V$. amygdalina leaves, transcriptomic libraries were constructed from 10, 20, and $30 \mathrm{DAG}$ and sequenced by illumina platform. More than $5 \mathrm{G}$ data were obtained from each sample, respectively. All clean reads generated from $V$. amygdalina leaves were assembled by the Trinity software, and the resulting 60,422 genes (N50: 1,625bp) were obtained. From the BLAST results, 26,766 (44.30\%), 31,723 (52.50\%), and 23,261 (38.50\%) genes were functionally annotated in TAIR, Nr, and SwissProt databases, respectively (Supplementary Table S3). $77.7 \%$ of BUSCO's core Eudicots ortholog genes (Simão et al. 2015) present in our assembly (Supplementary Table S4). These data provide abundant genetic resources of $V$. amygdalina for further studies.

\section{Analysis of differential gene expression}

To fully explore the differentially expressed genes in developing $V$. amygdalina, clean reads of each library were mapped into the generated gene database, and the expression levels were calculated by TPM. A total of 2,074 differentially expressed genes were identified (Supplementary Table S5). The results showed that 10:20 DAG, 10:30 DAG and 20:30 DAG had 1,115 (549 up-regulated and 566 downregulated genes), 1,619 (804 up-regulated and 815 down-regulated genes) and 467 (169 up-regulated and 298 down-regulated genes) differential genes, respectively (Supplementary Table S5). By Venn diagram analysis, 79, 246, and 205 were specific DEGs in 10:20, 10:30, and 20:30 DAG, respectively (Figure 3).

\section{Formation of malonyl-CoA}

ACL and ACC enzymes are responsible for generating the cytosolic pool of acetyl-CoA and malonylCoA, respectively. Three $A C L \alpha(V a-A C L \alpha 1, V a-A C L \alpha 2$, and $V a-A C L \alpha 3)$, one $A C L \beta(V a-A C L \beta 2)$, and one ACC $(V a-A C C l)$ genes were annotated in $V$. amygdalina (Supplementary Table S6). Abundant transcript levels of $V a-A C L \alpha 1, V a-A C L \alpha 3, V a-A C L \beta 2$, and $V a-A C C 1$ were present in developing $V$. amygdalina leaves, whereas $\mathrm{Va}-\mathrm{ACL} \alpha 2$ was expressed at low levels (Figure 4). Interestingly, the expressions of $\mathrm{Va}$ $A C L \alpha 1, V a-A C L \alpha 3, V a-A C L \beta 2$, and $V a-A C C 1$ all showed a coordinated profile with the highest expression at $10 \mathrm{DAG}$ (Figure 4).

\section{Formation of $p$-coumaroyl-CoA}

PAL is the first committed enzyme in the phenylalanine pathway to provide substrate for flavonoid biosynthesis. In this study, three homologous $P A L$ genes were obtained from the $V$. amygdalina transcriptome database (Supplementary Table S6). By alignment and phylogenetic analysis with $P A L$ genes from Lactuca sativa, the results showed that these $P A L$ genes were conserved (Supplementary Figure $\mathrm{S} 2$ and $\mathrm{S} 3$ ). The three $V a-P A L$ genes all showed highest expressions at $10 \mathrm{DAG}$, among which the expression levels of $V a-P A L 4$ and $V a-P A L 1$ were significantly more abundant than $V a-P A L 2$ in developing $V$. amygdalina leaves (Figure 4 and 5).

$\mathrm{C} 4 \mathrm{H}$ is a cytochrome $\mathrm{P} 450$ monooxygenase, involving in the hydroxylation at $\mathrm{C}-4$ position of transcinnamic acid. By functional annotation, two homologous $\mathrm{C} 4 \mathrm{H}$ genes were identified in $V$. amygdalina (Supplementary Table S6). It is also worth noting that, although both of them had a similar expression pattern in developing $\mathrm{V}$. amygdalina leaves, the $\mathrm{Va}-\mathrm{C} 4 \mathrm{H} 1$ expression was higher than $\mathrm{Va}-\mathrm{C} 4 \mathrm{H} 2$ (Figure 4).

Peer] reviewing PDF | (2020:11:55707:2:0:NEW 8 Mar 2021) 
290

291

292

293

294

295

296

297

298

299

300

301

\section{2}

$4 \mathrm{CL}$ enzyme catalyzes the CoA-activation of $p$-coumaric acid to form $p$-coumaroyl CoA. Four isoforms of $V a-4 C L$ genes were identified in $V$. amygdalina (Supplementary Table S6). Of these, expression levels for $\mathrm{Va}-4 C L 3$ were the most abundant isoform in developing $\mathrm{V}$. amygdalina leaves (Figure 4). Moreover, $\mathrm{Va}-4 C L 3$ gene exhibited high expressions at 10 and 20 DAG (Figure 4 and 5). The central flavonoid biosynthetic pathway

CHS is the first committed enzyme in all flavonoid biosynthesis. Two CHS genes, $\mathrm{Va}-\mathrm{CHSI}$ and $\mathrm{Va}$ CHS3 are identified from $V$. amygdalina leaves (Supplementary Table S6). Although both of them exhibited the highest expression at $10 \mathrm{DAG}, \mathrm{CHS1}$ gene displayed several-fold higher expression in comparison to $C H S 3$ gene during the development of $V$. amygdalina leaves (Figure 4).

$\mathrm{CHI}$ catalyzes the stereospecific cyclization of naringenin chalcone to (2S)-naringenin. Only one $\mathrm{Va}$ CHI gene was identified from $V$. amygdalina leaves (Supplementary Table S6). Similar to the expression patterns for $\mathrm{Va}-\mathrm{CHSl}$ genes, $\mathrm{Va}-\mathrm{CHI}$ gene showed the highest expressions at $10 \mathrm{DAG}$ (Figure 4 and 5).

Interestingly, once flavanones have been formed, they are then converted into different flavonoid kinds by different regulatory enzymes. FNSII catalyzes the conversion of flavanones to flavones, while $\mathrm{F} 3 \mathrm{H}$ and IFS are responsible for the conversion of flavanones to isoflavones and dihydroflavonols (Figure 4), respectively. One $V a-F N S I I$ and one $V a-F 3 H$ genes were identified in $V$. amygdalina leaves, and displayed an abundant transcript at $10 \mathrm{DAG}$ (Figure 4 and 5). Three $\mathrm{Va}$-IFS genes were found in this work, of which $V a-I F S 2$ had a high expression at $10 \mathrm{DAG}$, and the expressions of $V a-I F S 3$ showed an increase with the leaf development (Figure 4).

FLS functions in the biosynthesis of flavonols. Three homologous $V a-F L S$ genes were obtained (Supplementary Table S6). During developing V. amygdalina leaves, Va-FLS1 and Va-FLS2 genes showed up-regulated expressions at $20 \mathrm{DAG}$, whereas $V a-F L S 3$ gene displayed the highest expression level at 10 DAG (Figure 4).

Flavonoid 3',5'-hydroxylase (F3'5'H) belongs to the cytochrome P-450 family. Although hundreds of genes were annotated as P-450 family (Supplementary Table S3), only one gene was identified as $\mathrm{Va}$ F3'5' $\mathrm{H}$ gene involved in 3'- or/and 5'-hydroxylation of flavonoids by KEGG analysis (Supplementary Table S6 and Supplementary Table S6). Similar to the above genes involved in the central flavonoid biosynthetic pathway, the $V a-F 3^{\prime} 5^{\prime} H$ gene also showed the highest expression level at 10 DAG (Figure 4 and 5).

\section{Tailoring reactions of flavonoid scaffolds}

According to the above LC-MS results, the possible modification pathway of flavonoid biosynthesis was constructed (Figure 6a), reflecting glycosylation and methylation were common modifications in $V$. amygdalina leaves. Based on the homology with known genes of $A$. thaliana involved in glycosylation, methylation, and acetylation of flavonoids, a total of 46, 9, and 18 genes potentially encoding flavonoid glycosyltransferase (FGT), flavonoid methyltransferase (FMT), and flavonoid acyltransferase (FAT) were identified, respectively (Supplementary Table S7). These gene expressions showed distinctly temporal patterns in developing $V$. amygdalina leaves, suggesting that they may function in different development for the metabolite modifications. Notably, correlation analysis indicated that the expression levels of 12 $\mathrm{Va}-F G T$ and $4 \mathrm{Va}-F M T$ genes showed a positive correlation with the relative contents of some glycosylated and methylated flavonoids during developing $V$. amygdalina leaves, respectively (Figure 6b, 6c, and Supplementary Table S8). These expression results suggested a possible involvement of the correlated genes in glycosylation or methylation of flavonoids.

\section{Discussion}


303

304

305

306

307

308

309

310

311

312

313

314

315

316

317

318

319

320

321

322

323

324

325

326

327

328

329

330

331

332

333

334

335

336

337

338

339

340

341

342

343

344

345

346

Because of various pharmacological properties in $V$. amygdalina leaves, the plant has been widely used in diet and medicine in Africa and Asia (Yeap et al. 2010). Previous studies of $V$. amygdalina mainly focused on biological activity and extraction method, indicating that flavonoids are abundant in leaves (Alara et al. 2018; Ayoola et al. 2008; Igile et al. 1994). However, it is lack of systematical investigation on flavonoid profiles and biosynthetic pathway in developing $V$. amygdalina leaves. During the development of $V$. amygdalina leaves, the flavonoid components were qualitatively and quantificationally analyzed in this study. A total of 42 flavonoids were identified in $V$. amygdalina leaves, mainly including flavones, dihydroflavones, isoflavones, and flavonols (Supplementary Table S2). These results verify that $V$. amygdalina leaves possess abundant flavonoids, reflecting that $V$. amygdalina is a natural source of flavonoids (Atangwho et al. 2013).

A lack of genome information in $V$. amygdalina remains a large obstacle to comprehensively understanding biosynthetic pathway of flavonoids. In an effort to overcome this limitation, we constructed transcriptomic libraries of $V$. amygdalina leaves at 10,20, and $30 \mathrm{DAG}$, and sequenced by Illumina HiSeqTM platform. The resulting 60,422 genes were assembled and obtained, and a total of 18,863 reliable unigenes $(\geq 1000$ bp) identified in $V$. amygdalina leaves (Supplementary Table S3). In addition, 31,872 genes were annotated at least in one of public databases (Supplementary Table S3) and 2,074 differential expression genes were obtained (Figure 3). Thus, these molecular resources massively filled and enriched the gene dataset of $V$. amygdalina, which will contribute to advancing the research regarding comparative and functional genomics. Together, the LC-MS and transcriptomic data contribute to further explore on the pathway of flavonoid biosynthesis in $V$. amygdalina leaves (Figure 4 and 6).

In plants. acetyl-CoA is an important carbon source for the biosynthesis of various metabolites, such as fatty acids, flavonoids, and terpenoids (Feng et al. 2020). Previous study indicated that the molecule of CoA moiety is too large to transport across biological membranes, therefore the required acetyl-CoA must be separately synthesized in different subcellular compartments (Brooks \& Stumpf 1966). Notably, flavonoid biosynthesis occurs in cytoplasm, and ACL is now known to the ATP-dependent enzyme responsible for generating the cytosolic acetyl-CoA. It was reported that the ACL heteromer is composed of ACL $\alpha$ and ACL $\beta$ (Fatland et al. 2002). As expected, three $A C L \alpha(V a-A C L \alpha 1, V a-A C L \alpha 2$, and $V a$ $A C L \alpha 3$ ) and one $A C L \beta(V a-A C L \beta 2)$ genes was identified in $V$. amygdalina leaves (Supplementary Table S6). Expression analysis in developing $V$. amygdalina leaves indicated a closely coordinated expression between $V a-A C L \alpha 1 / 3$ and $V a-A C L \beta 2$ (Figure 4). Thus, it is tempting to speculate that the combination of $V a-A C L \alpha 1 / 3$ and $V a-A C L \beta 2$ may be critical for ACL activity in $V$. amygdalina leaves, as in previous study that only $A C L \alpha$ or $A C L \beta$ transcript would lead to a defect in ACL function (Fatland \& Wurtele 2005).

Once the acetyl-CoA has been produced in cytoplasm, ACC can catalyze the carboxylation of acetylCoA into malonyl-CoA that is shared between flavonoid biosynthesis and the elongation of very long chain fatty acids ( $C \geq 20)$ (Alban et al. 2000). Previous studies have confirmed that $A$. thaliana has three ACC isozymes, of which a heteromeric form is composed of four distinct subunits (At5g16390, At5g35360, At2g38040, and AtCg00500) located in plastids, while other two heteromeric forms of ACC1 (At1g36160) and ACC2 (At1g36180) comprise a large polypeptide located in cytoplasm and chloroplast, respectively ( $\mathrm{Li}$ et al. 2011). Thus, ACC1 is responsible for the generation of cytosolic malonyl-CoA for flavonoid biosynthesis in $A$. thaliana (Saito et al. 2013). Indeed, one obvious homolog ( Va-ACCl) of At$A C C 1$ was found in $V$. amygdalina leaves (Supplementary Table S6), and $V a-A C C 1$ gene was coexpressed with $V a-A C L \alpha 1 / 3$ and $V a-A C L \beta 2$ genes (Figure 4). Thus, $V a-A C C 1$ may control the carbon flux into malonyl-CoA for flavonoid biosynthesis in $V$. amygdalina leaves. 
In addition to three molecules of malonyl-CoA, the flavonoid biosynthesis also needs one molecule of $p$-coumaroyl CoA. The formation of $p$-coumaroyl CoA must be orderly catalyzed by PAL, $\mathrm{C} 4 \mathrm{H}$, and $4 \mathrm{CL}$ enzymes (Ferreyra et al. 2012). Although there were three PAL isoforms, the expression levels of $\mathrm{Va}$ $P A L 1$ and $V a-P A L 4$ were the most abundant isoforms in developing $V$. amygdalina leaves (Figure 4). Thus, they may partially complement the function of each other in conversion of phenylalanine to transcinnamic, as was reported in A. thaliana (Ohl et al. 1990). Two orthologs ( $\mathrm{Va}-\mathrm{C} 4 \mathrm{HI}$ and $\mathrm{Va}-\mathrm{C} 4 \mathrm{H} 2$ ) of $\mathrm{A}$. thaliana $\mathrm{C} 4 \mathrm{H}$ (At2g30490) were characterized in this study, but the expressions of $\mathrm{Va}$ - $\mathrm{C} 4 \mathrm{H} 1$ were 5 times higher than that of $\mathrm{Va}-\mathrm{C} 4 \mathrm{H} 2$ (Supplementary Table S6). Our data suggest that Va-C4H1 is presumed to represent the principal isoform responsible for the formation of $p$-coumaric acid in $V$. amygdalina leaves. Among the four 4CL isoforms, only the $\mathrm{Va}-4 C L 3$ gene was homologous with At-4CL3 (At1g65060), and showed the highest expression than the other three $\mathrm{Va}-4 C L$ genes (Supplementary Table S6). These data imply that Va-4CL3 may be the major enzyme catalyzing $p$-coumaroyl-CoA production, in agreement with the previous view in $A$. thaliana (Ehlting et al. 1999). Together, the expression levels of the above interested genes showed a pattern that correlated with the biosynthesis of their corresponding production (Figure 4 and 5). Thus, they may collaborate to facilitate the $p$-coumaroyl-CoA production destined to flavonoid biosynthesis in $V$. amygdalina leaves.

As a type III polyketide synthase enzyme, CHS catalyzes the Claisen-ester condensation that is the first committed rate-limiting step in the flavonoid biosynthesis (Austin \& Noel 2003). Here, two CHS isoforms of $\mathrm{Va}$-CHSI and $\mathrm{Va}$-CHS3 were the orthologs with A. thaliana At-CHS (At5g13930), which has been verified to be involved in first Claisen-ester condensation of flavonoid biosynthesis (Shirley et al. 1995). The expression levels of $\mathrm{Va}$-CHS1 gene were more abundant than $\mathrm{Va}$-CHS3 gene in developing $\mathrm{V}$. amygdalina leaves, suggesting a crucial role of $\mathrm{Va}-\mathrm{CHSl}$ in the synthesis of naringenin chalcone. Additionally, the homology of $A t-C H I$ (At3g55120) that participates in stereospecific cyclization for (2S)naringenin formation (Saito et al. 2013) was found in $\mathrm{V}$. amygdalina leaves as $\mathrm{Va}$-CHI. Interestingly, the expressions of $\mathrm{Va}-\mathrm{CHI}$ gene were comparable to that of $\mathrm{Va}$-CHSI gene (Figure 5) and displayed a coordinated temporal pattern with the naringenin accumulation in developing $V$. amygdalina leaves (Figure 4). Thus, Va-CHI enzyme is a possible candidate related to (2S)-naringenin biosynthesis in $V$. amygdalina leaves.

It is notable that the generated flavanones would have different fates catalyzed by different regulatory enzymes (Figure 4). One of pathway is flavone biosynthesis catalyzed by FNS, which dehydrogenize flavanones into flavones at $\mathrm{C} 2$ and $\mathrm{C} 3$ positions. In plants, there are two distinct enzyme systems of FNS I and FNS II, of which FNS I is mainly distributed in the Apiaceae family (Martens et al. 2001), while FNS II widely distributed in many families of higher plants (Wu et al. 2016). Sure enough, only one Va-FNSII gene was identified in $V$. amygdalina leaves, and its expression exhibit a similar pattern with apigenin contents in developing $V$. amygdalina leaves (Figure 4). Thus, Va-FNSII may be the key enzyme responsible for flavone formation in $V$. amygdalina leaves. In addition, there is another pathway of isoflavone biosynthesis, in which IFS catalyzes the transference of aromatic group from 2-position to 3-position (Misra et al. 2010). Three Va-IFS isoforms were found in $V$. amygdalina leaves (Supplementary Table S6). Notably, the expression levels of $V a-I F S 3$ showed a pattern that correlated with genistein biosynthesis (Figure 4), implying an important role of Va-IFS3 in this metabolic process.

F3H and FLS catalyze the last branch of the trunk pathway towards flavonol biosynthesis (Figure 4). Although the homology of At-F3H (At3g51240) was found in V. amygdalina leaves, its expression was either expressed at low levels or absent (Figure 4). Thus, it is tempting to speculate that Va-IFS3 may be not essential for the oxygenation at 3-postion of flavanone. Previous studies in A. thaliana decided that 
391

392

393

394

395

396

397

398

399

400

401

402

403

404

405

406

407

408

409

410

411

412

413

414

415

416

417

418

419

420

421

422

423

424

425

426

427

428

429

430

431

432

433

434

the activity of $\mathrm{F} 3 \mathrm{H}$ in vivo can be partially compensated by two related 2-oxoglutarate-dependent dioxygenases (2-OODs) (Pelletier \& Shirley 1996a). One of the 2-ODD enzymes is FLS, catalyzing the double bond formation between C-2 and C-3 position of flavonols (Pelletier \& Shirley 1996a; Prescott et al. 2002). Three Va-FLS isoforms were identified (Supplementary Table S6), and showed distinct expression profiles in developing $V$. amygdalina leaves (Figure 4). These expression results, coupled with either lack or low content in kaempferol and quercetin (Supplementary Table S2), suggests that Va-FLS may have other functions in flavonoid biosynthesis. This view also was supported by the detailed mechanistic study in A. thaliana (Turnbull et al. 2004). Intriguingly, due to lack of intermediate metabolite of dihydrokaempferol in $V$. amygdalina leaves, how kaempferol is formed excited our interest. We observed a tight connection of molecular structure between kaempferol and apigenin (Figure 4), and their contents showed a coordinated temporal pattern in developing $V$. amygdalina leaves (Supplementary Table S6). Thus, it is hypothesized whether flavone 3-hydroxylase exist in $V$. amygdalina leaves. These data provide leads for future research on the interconnection of pathways involved in flavones and flavonols.

\section{Conclusions}

In conclusion, metabolome analysis indicated abundant and diverse flavonoids in $V$. amygdalina leaves, including 6 dihydroflavones, 14 flavones, 8 isoflavones, 9 flavonols, 2 xanthones, 1 chalcone, 1 cyanidin, and 1 dihydroflavonol. Additionally, glycosylation and methylation commonly occur at -OH moieties of the $\mathrm{C} 3$ and $\mathrm{C} 7$ positions of flavonoid aglycones. By transcriptome sequencing, 60,422 genes were assembled, among which 31,872 genes were annotated at least in one of public databases. These sequencing results massively filled in the blanks in gene dataset of $V$. amygdalina. The temporal and comparative combination of metabolome and transcriptome results points to the key genes encoding regulatory enzymes involved in material supplying, flavonoid scaffold biosynthesis, and flavonoid modifications. These findings in the present study would be conducive to understand the underlying mechanisms of flavonoid biosynthesis in $V$. amygdalina.

\section{Acknowledgements} -

\section{References}

Alara OR, Abdurahman NH, and Olalere OA. 2018. Optimization of microwave-assisted extraction of flavonoids and antioxidants from Vernonia amygdalina leaf using response surface methodology. Food Bioprod Process 107:36-48.

Alara OR, Abdurahman NH, and Olalere OA. 2020. Ethanolic extraction of flavonoids, phenolics and antioxidants from Vernonia amygdalina leaf using two-level factorial design. J King Saud Univ Sci 32:7-16.

Alban C, Job D, and Douce R. 2000. Biotin metabolism in plants. Annu Rev Plant Biol 51:17-47.

Asante DB, Effah-Yeboah E, Barnes P, Abban HA, Ameyaw EO, Boampong JN, Ofori EG, and Dadzie JB. 2016. Antidiabetic effect of young and old ethanolic leaf extracts of Vernonia amygdalina: A comparative study. $J$ Diabetes Res 2016:1-13.

Atangwho IJ, Egbung GE, Ahmad M, Yam MF, and Asmawi MZ. 2013. Antioxidant versus anti-diabetic properties of leaves from Vernonia amygdalina Del. growing in Malaysia. Food Chem 141:3428-3434.

Austin MB, and Noel JP. 2003. The chalcone synthase superfamily of type III polyketide synthases. Nat Prod Rep 20:79-110. 
435

436

437

438

439

440

441

442

443

444

445

446

447

448

449

450

451

452

453

454

455

456

457

458

459

460

461

462

463

464

465

466

467

468

469

470

471

472

473

474

475

476

477

478

479

480

481

482

483

484

485

486

487

488

489

Ayoola G, Coker H, Adesegun S, Adepoju-Bello A, Obaweya K, Ezennia EC, and Atangbayila T. 2008. Phytochemical screening and antioxidant activities of some selected medicinal plants used for malaria therapy in Southwestern Nigeria. Trop J Pharm Res 7:1019-1024.

Brooks JL, and Stumpf P. 1966. Fat metabolism in higher plants: XXXIX. Properties of a soluble fatty acid synthesizing system from lettuce chloroplasts. Arch Biochem Biophys 116:108-116.

Dixon RA, and Pasinetti GM. 2010. Flavonoids and isoflavonoids: from plant biology to agriculture and neuroscience. Plant Physiol 154:453-457.

Ehlting J, Büttner D, Wang Q, Douglas CJ, Somssich IE, and Kombrink E. 1999. Three 4-coumarate: coenzyme A ligases in Arabidopsis thaliana represent two evolutionarily divergent classes in angiosperms. Plant J 19:920.

Erasto P, Grierson DS, and Afolayan AJ. 2007. Antioxidant constituents in Vernonia amygdalina Leaves. Pharm Biol 45:195-199.

Fatland BL, Ke J, Anderson MD, Mentzen WI, Cui LW, Allred CC, Johnston JL, Nikolau BJ, and Wurtele ES. 2002. Molecular characterization of a heteromeric ATP-citrate lyase that generates cytosolic acetylcoenzyme A in Arabidopsis. Plant Physiol 130:740-756.

Fatland BL, and Wurtele NES. 2005. Reverse Genetic Characterization of Cytosolic Acetyl-CoA Generation by ATP-Citrate Lyase in Arabidopsis. Plant Cell 17:182-203.

Feng X, Zhang L, Xu S, and Shen A-z. 2020. ATP-citrate lyase (ACLY) in lipid metabolism and atherosclerosis: An updated review. Prog Lipid Res 77:101006.

Ferreyra F, Lorena M, Rius S, and Casati P. 2012. Flavonoids: biosynthesis, biological functions, and biotechnological applications. Front Plant Sci 3:222.

Ferreyra MLF, Emiliani J, Rodriguez EJ, Campos-Bermudez VA, Grotewold E, and Casati P. 2015. The identification of maize and Arabidopsis type I flavone synthases links flavones with hormones and biotic interactions. Plant Physiol 169:1090-1107.

Götz S, Garcíagómez JM, Terol J, Williams TD, Nagaraj SH, Nueda MJ, Robles M, Talón M, Dopazo J, and Conesa A. 2008. High-throughput functional annotation and data mining with the Blast2GO suite. Nucleic Acids Res 36:3420-3435.

Hamberger B, and Hahlbrock K. 2004. The 4-coumarate: CoA ligasegene family in Arabidopsis thaliana comprises one rare, sinapate-activating and three commonly occurring isoenzymes. P Natl Acad Sci USA 101:22092214.

Igile GO, Oleszek W, Jurzysta M, Burda S, Fafunso M, and Fasanmade AA. 1994. Flavonoids from Vernonia amygdalina and their antioxidant activities. J Agr Food Chem 42:2445-2448.

Jung W, Yu O, Lau SMC, O"Keefe DP, Odell J, Fader G, and Mcgonigle B. 2000. Identification and expression of isoflavone synthase, the key enzyme for biosynthesis of isoflavones in legumes. Nat Biotechnol 18:208212.

Kanehisa M, Araki M, Goto S, Hattori M, Hirakawa M, Itoh M, Katayama T, Kawashima S, Okuda S, and Tokimatsu T. 2008. KEGG for linking genomes to life and the environment. Nucleic Acids Res 36:D480D484.

Lepiniec L, Debeaujon I, Routaboul J-M, Baudry A, Pourcel L, Nesi N, and Caboche M. 2006. Genetics and biochemistry of seed flavonoids. Annu Rev Plant Biol 57:405-430.

Li D, Chen G, Ma B, Zhong C, and He N. 2020. Metabolic Profiling and Transcriptome Analysis of Mulberry Leaves Provide Insights into Flavonoid Biosynthesis. J Agr Food Chem 68:1494-1504.

Li X, Ilarslan H, Brachova L, Qian H-R, Li L, Che P, Wurtele ES, and Nikolau BJ. 2011. Reverse-genetic analysis of the two biotin-containing subunit genes of the heteromeric acetyl-coenzyme A carboxylase in Arabidopsis indicates a unidirectional functional redundancy. Plant Physiol 155:293-314.

Li Y, Baldauf S, Lim E-K, and Bowles DJ. 2001. Phylogenetic analysis of the UDP-glycosyltransferase multigene family of Arabidopsis thaliana. J Biol Chem 276:4338-4343.

Liu C, Yu Q, Li Z, Jin X, and Xing W. 2020. Metabolic and transcriptomic analysis related to flavonoid biosynthesis during the color formation of Michelia crassipes tepal. Plant Physiol Bioch 155:938-951.

Maeda H, and Dudareva N. 2012. The Shikimate Pathway and Aromatic Amino Acid Biosynthesis in Plants. Annu Rev Plant Biol 63:73.

Martens S, Forkmann G, Matern U, and Lukačin R. 2001. Cloning of parsley flavone synthase I. Phytochemistry 58:43-46.

Misra P, Pandey A, Tewari SK, Nath P, and Trivedi PK. 2010. Characterization of isoflavone synthase gene from Psoralea corylifolia: a medicinal plant. Plant Cell Rep 29:747-755. 
490

491

492

493

494

495

496

497

498

499

500

501

502

503

504

505

506

507

508

509

510

511

512

513

514

515

516

517

518

519

520

521

522

523

524

525

526

527

528

529

530

531

532

533

534

535

Nwanjo HU. 2005. Efficacy of aqueous leaf extract of Vernonia amygdalina on plasma lipoprotein and oxidative status in diabetic rat models. Nig J Physiol Sci 20:39-42.

Ohl S, Hedrick SA, Chory J, and Lamb CJ. 1990. Functional properties of a phenylalanine ammonia-lyase promoter from Arabidopsis. Plant Cell 2:837-848.

Pelletier MK, and Shirley BW. 1996a. Analysis of flavanone 3-hydroxylase in Arabidopsis seedlings -Coordinate regulation with chalcone synthase and chalcone isomerase. Plant Physiol 111:339-345.

Pelletier MK, and Shirley BW. 1996b. Analysis of Flavanone 3-Hydroxylase in Arabidopsis Seedlings (Coordinate Regulation with Chalcone Synthase and Chalcone Isomerase). Plant Physiology 111:339-345.

Prescott AG, Stamford NP, Wheeler G, and Firmin JL. 2002. In vitro properties of a recombinant flavonol synthase from Arabidopsis thaliana. Phytochemistry 60:589-593.

Ramakers C, Ruijter JM, Deprez RH, and Moorman AF. 2003. Assumption-free analysis of quantitative real-time polymerase chain reaction (PCR) data. Neuroscience Lett 339:62-66.

Saito K, Yonekura-Sakakibara K, Nakabayashi R, Higashi Y, Yamazaki M, Tohge T, and Fernie AR. 2013. The flavonoid biosynthetic pathway in Arabidopsis: structural and genetic diversity. Plant Physiol Bioch 72:2134.

Schilmiller AL, Stout J, Weng JK, Humphreys J, and Chapple C. 2009. Mutations in the cinnamate 4-hydroxylase gene impact metabolism, growth and development in Arabidopsis. Plant J 60:771-782.

Shirley BW, Kubasek WL, Storz G, Bruggemann E, Koornneef M, Ausubel FM, and Goodman HM. 1995. Analysis of Arabidopsis mutants deficient in flavonoid biosynthesis. Plant J 8:659-671.

Silva-Navas J, Moreno-Risueno MA, Manzano C, Téllez-Robledo B, Navarro-Neila S, Carrasco V, Pollmann S, Gallego FJ, and Del Pozo JC. 2016. Flavonols mediate root phototropism and growth through regulation of proliferation-to-differentiation transition. Plant Cell 28:1372-1387.

Simão FA, Waterhouse RM, Ioannidis P, Kriventseva EV, and Zdobnov EM. 2015. BUSCO: assessing genome assembly and annotation completeness with single-copy orthologs. Bioinformatics 31:3210-3212.

Tohge T, De Souza LP, and Fernie AR. 2017. Current understanding of the pathways of flavonoid biosynthesis in model and crop plants. $J$ Exp Bot:15.

Tohge T, and Fernie AR. 2017. An overview of compounds derived from the shikimate and phenylpropanoid pathways and their medicinal importance. Mini-Rev Med Chem 17:1013-1027.

Tohge T, Wendenburg R, Ishihara H, Nakabayashi R, Watanabe M, Sulpice R, Hoefgen R, Takayama H, Saito K, and Stitt M. 2016. Characterization of a recently evolved flavonol-phenylacyltransferase gene provides signatures of natural light selection in Brassicaceae. Nat Commun 7:1-11.

Tona L, Cimanga RK, Mesia K, Musuamba CT, De Bruyne T, Apers S, Hernans N, Van Miert S, Pieters L, and Totté J. 2004. In vitro antiplasmodial activity of extracts and fractions from seven medicinal plants used in the Democratic Republic of Congo. J Ethnopharmacol 93:27-32.

Turnbull JJ, Nakajima J-i, Welford RW, Yamazaki M, Saito K, and Schofield CJ. 2004. Mechanistic studies on three 2-oxoglutarate-dependent oxygenases of flavonoid biosynthesis anthocyanidin synthase, flavonol synthase, and flavanone 3 $\beta$-hydroxylase. J Biol Chem 279:1206-1216.

Wu J, Wang X-C, Liu Y, Du H, Shu Q-Y, Su S, Wang L-J, Li S-S, and Wang L-S. 2016. Flavone synthases from Lonicera japonica and L. macranthoides reveal differential flavone accumulation. Sci Rep-UK 6:19245.

Yanai Y, Tsutomu K, Shimada H, Syrkin WE, Nikolau BJ, and Norio I. 1995. Genomic organization of $251 \mathrm{kDa}$ acetyl-CoA carboxylase genes in Arabidopsis: tandem gene duplication has made two differentially expressed isozymes. Plant Cell Physiol:779.

Yeap SK, Ho WY, Beh BK, Liang WS, Ky H, Yousr AHN, and Alitheen NB. 2010. Vernonia amygdalina, an ethnoveterinary and ethnomedical used green vegetable with multiple bioactivities. J Med Plants Res 4:2787-2812.

PeerJ reviewing PDF | (2020:11:55707:2:0:NEW 8 Mar 2021) 


\section{Figure 1}

Figure 1 The $V$. amygdalina leaves and flavonoid content at different development period.

Figure 1 The $V$. amygdalina leaves and flavonoid content at different development period. (a) The $V$. amygdalina leaves at different development period. (b) The total content of flavonoids in developing $V$. amygdalina leaves. Data are reported as mean \pm standard deviation. Different letters mean significant difference $(p<0.01)$.

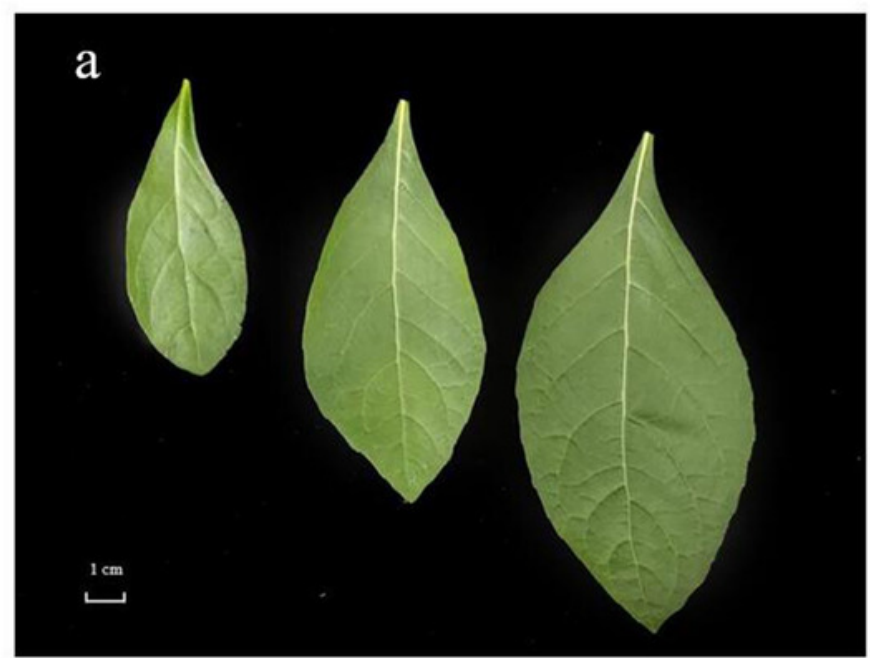

b

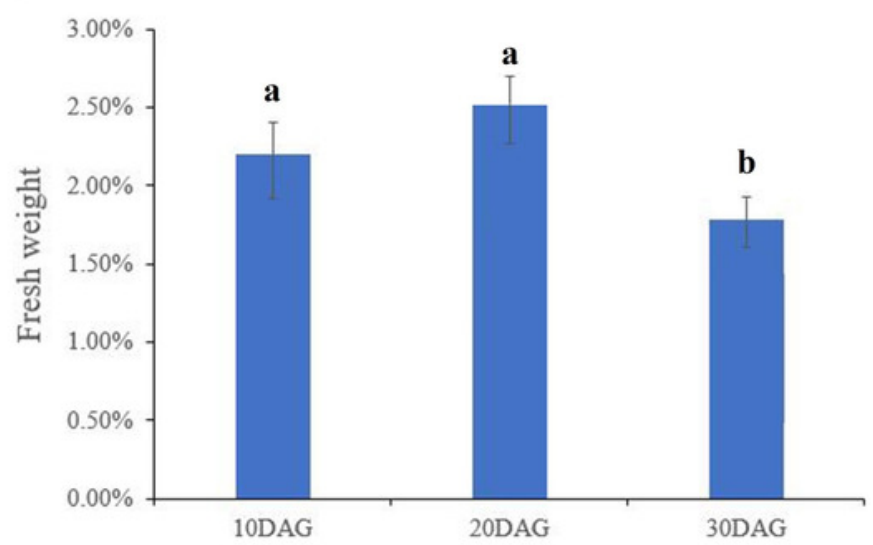




\section{Figure 2}

Figure 2 Heatmap of flavonoid profiles in Vernonia amygdalina leaves.

Figure 2 Heatmap of flavonoid profiles in Vernonia amygdalina leaves. The data were calculated as $\log _{10}($ Peak area+0.01). TCA, trans-cinnamic acid; 6-HF, 6Hydroxyflavanone; 6,9-DRNA, 6,8-Diprenylnaringenin; 2'-HF, 2'-Hydroxyflavanone; 8MButDMPCr, 8-(2,3-Dihydroxy-3-methylbutyl)-5,7-dimethoxy-2-phenyl-2,3-dihydro-4Hchromen-4-one; 7-HF, 7-Hydroxyflavone; 3'-HF, 3'-Hydroxyflavone; HPCA- $\beta$-D-Glc, 5Hydroxy-2-(4-hydroxyphenyl)-4-oxo-4H-chromen-7-yl 6-0-acetyl- $\beta$-D-glucopyranoside; MOPC$\beta$-D-GlcA, 5-Hydroxy-2-(3-hydroxy-4-methoxyphenyl)-4-oxo-4H-chromen-7-yl $\beta$-Dglucopyranosiduronic acid; BA- $\beta$-D-G, Biochanin A- $\beta$-D-glucoside; IF O-G, Isoflavonoid Oglycosides; KF 7-O- $\beta$-D-Glc, kaempferol 7-O- $\beta$-D-glucopyranoside; KF-3-GICA, Kaempferol-3Glucuronide; $\alpha$-L-GHCHPEH, 5-[3-( $\alpha$-L-glycero-Hexopyranosyloxy)-5,7-dihydroxy-4-oxo-4Hchromen-2-yl]-2,3-dihydroxyphenyl $\alpha$-L-erythro-hexopyranoside; NC, Naringenin chalcone; 7HF, 7-hydroxyflavonoids. 


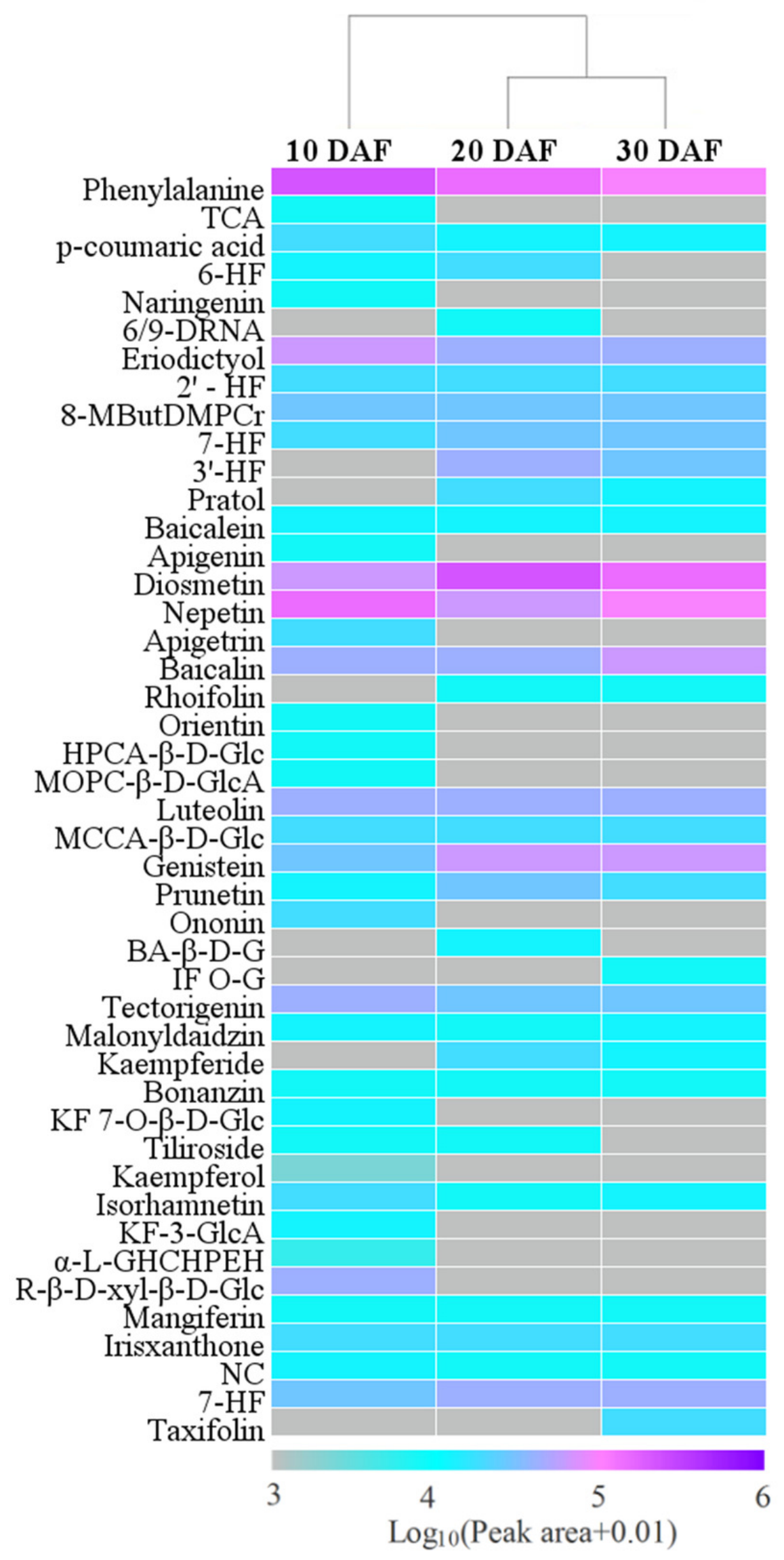


Figure 3

Figure 3 Number and distribution of differently expressed genes.

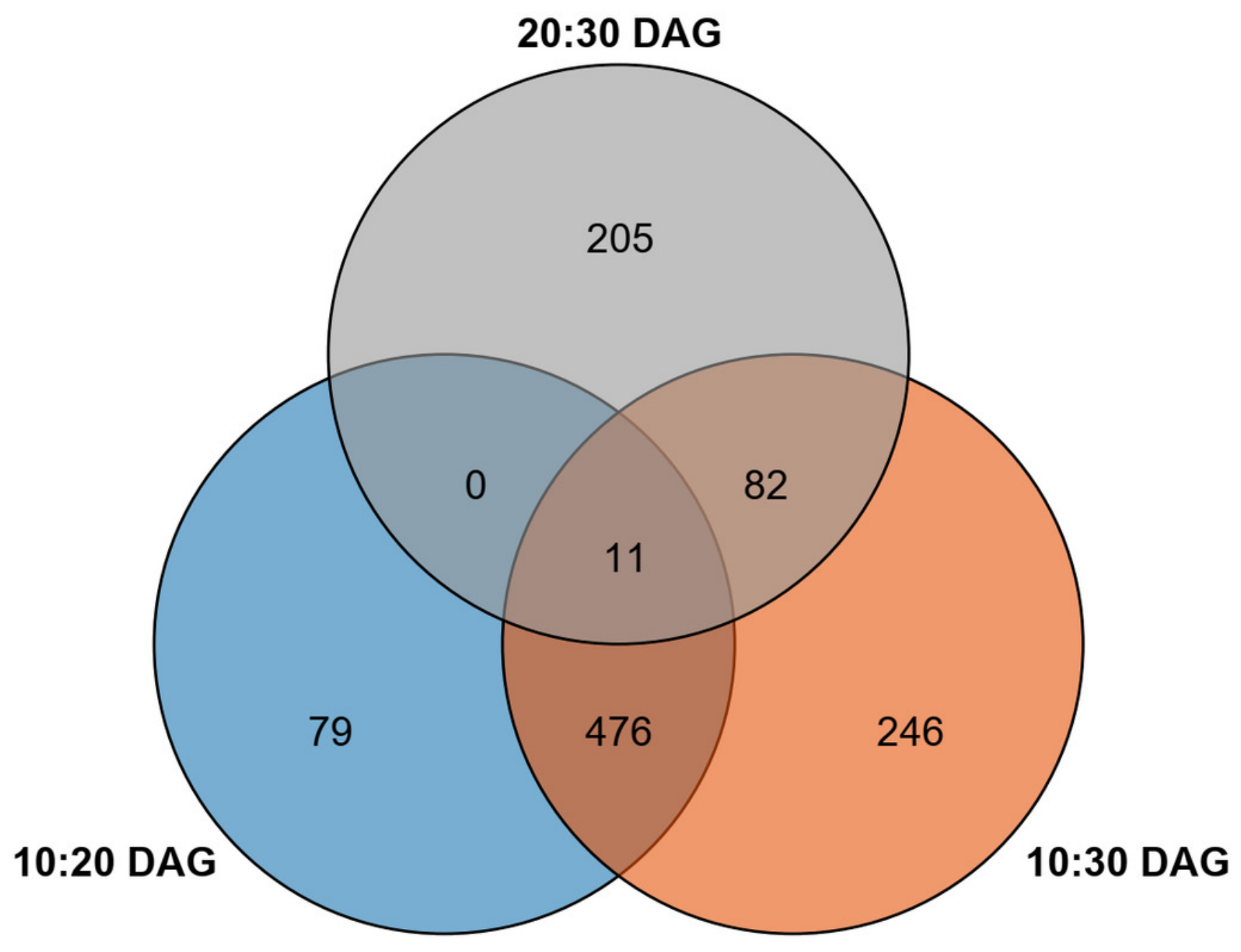


Figure 4

Figure 4 Biosynthetic pathway of phenylpropanoid and flavonoid skeletons in Vernonia amygdalina leaves.

Figure 4 Biosynthetic pathway of phenylpropanoid and flavonoid skeletons in Vernonia amygdalina leaves. The heatmap from left to right represent the data from 10 DAG, 20 DAG, and 30 DAG, respectively. 


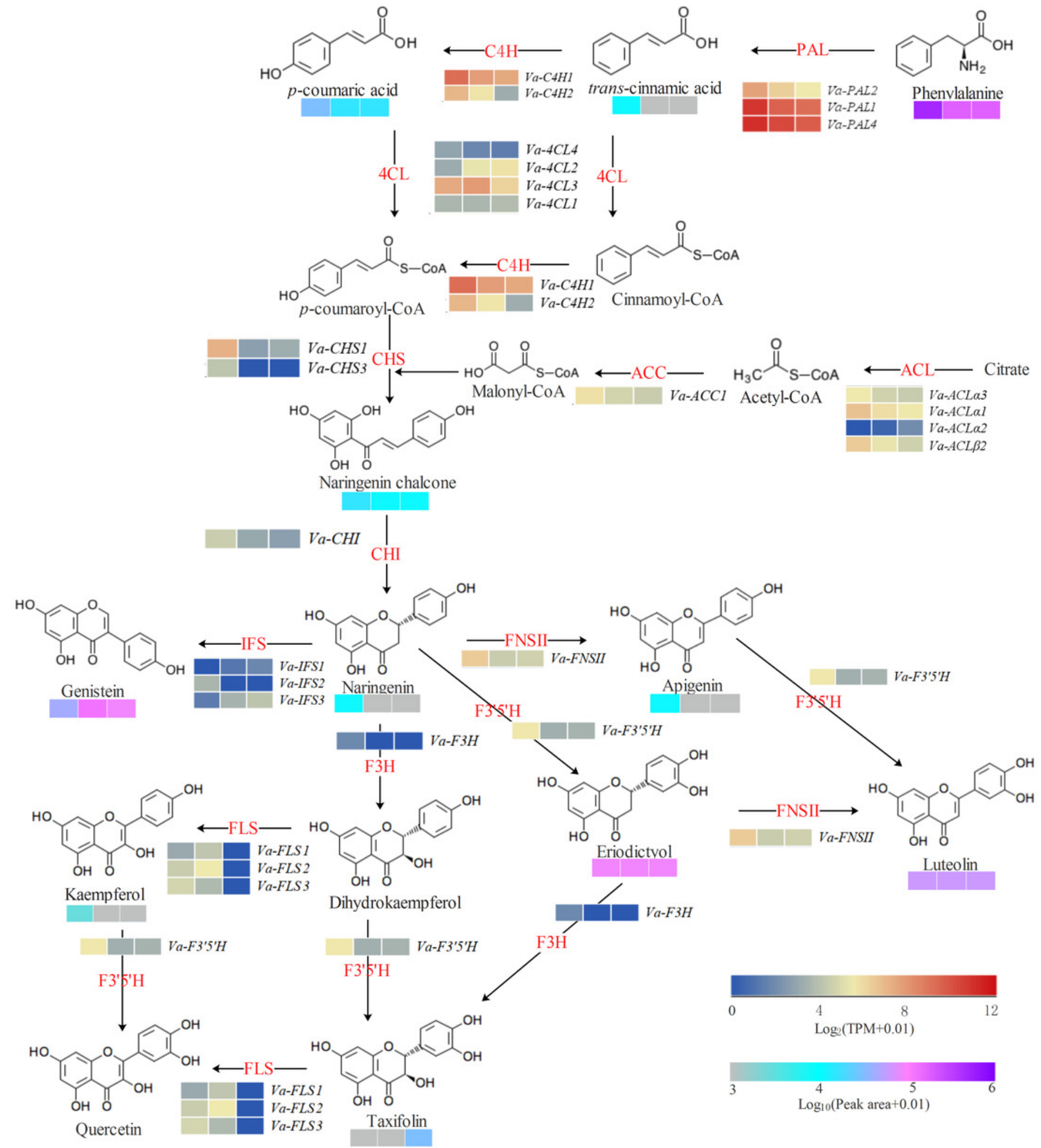


Figure 5

Figure 5 qRT-PCR analysis of potential genes involved in flavonoid biosynthesis.

Figure 5 qRT-PCR analysis of potential genes involved in flavonoid biosynthesis.

The statistical analysis was performed using Duncan method. Data are reported as mean \pm standard deviation. Different letters mean significant difference $(p<0.01)$.

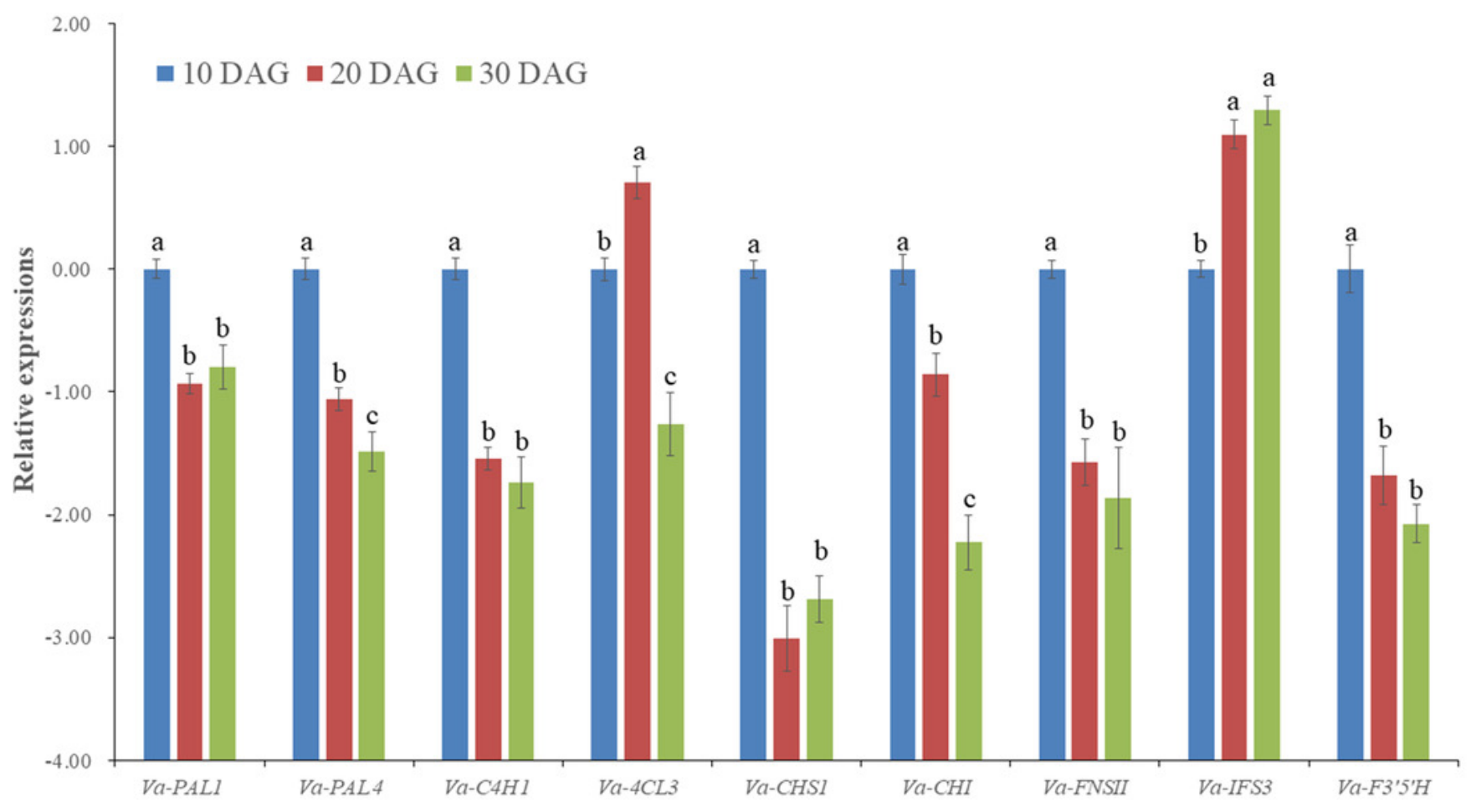


Figure 6

Figure 6 Flavonoid modifications in Vernonia amygdalina leaves.

Figure 6 Flavonoid modifications in Vernonia amygdalina leaves. (a) Proposed pathways of flavonoid modifications. Dotted lines represent this modification pathway needs two steps to complete. (b) These Va-FGT genes showed significantly positive correlation with the contents of glycosylated flavonoids in developing $V$. amygdalina leaves. (c) These Va-FMT genes showed significantly positive correlation with the contents of methylated flavonoids in developing $V$. amygdalina leaves.

$\mathbf{a}$
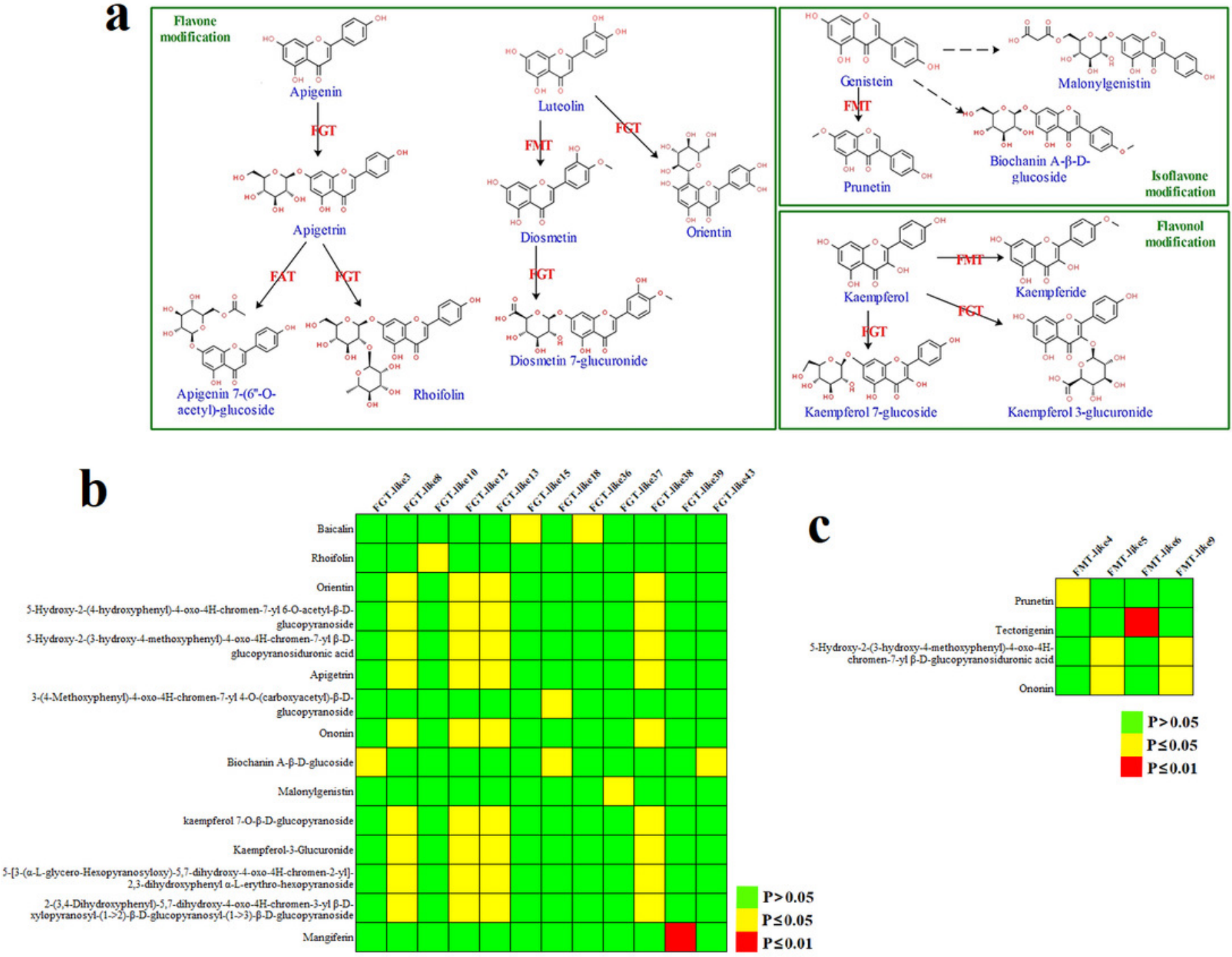